\title{
A novel 2 bp deletion variant in Ovine-DRB1 gene is associated with increased Visna/maedi susceptibility in Turkish sheep
}

\author{
Yalçın Yaman ( $\square$ yalcinyaman@gmail.com ) \\ Sheep Breeding and Research Institute \\ Veysel Bay \\ Eagean Agricultural Research Institute \\ Ramazan Aymaz \\ Sheep Breeding and Research Institute \\ Murat Keleş \\ Sheep Breeding and Research Institute \\ Yasemin Öner \\ Uludag University \\ Eden Yitna Teferedegn \\ Ege University \\ Cemal Ün \\ Ege University
}

\section{Research Article}

Keywords: Visna/Maedi (VM), sheep, TMEM154 gene, animal breeding

Posted Date: April 7th, 2021

DOI: https://doi.org/10.21203/rs.3.rs-387202/v1

License: (c) (i) This work is licensed under a Creative Commons Attribution 4.0 International License. Read Full License

Version of Record: A version of this preprint was published at Scientific Reports on July 14th, 2021. See the published version at https://doi.org/10.1038/s41598-021-93864-8. 


\section{Abstract}

Visna/Maedi (VM) is a multisystemic lentivirus infection of sheep that affecting sheep industry across the globe. TMEM154 gene has been identified to be a major VM associated host gene, nevertheless, a recent study showed that the frequency of the VM-resistant TMEM154 haplotypes was very low or absent in indigenous sheep. Thus, the present study was designed to determine other possible co-receptors associated with VM. For this purpose, $D R B 1$ gene, which is renowned for its role in host immune response activity against various diseases was targeted. A total number of 151 case-control matched pairs were constructed from 2266 ELISA tested sheep. A broad range of DRB1 haplotype diversity was detected by sequence-based genotyping. Moreover, a novel 2 bp deletion (del) in the DRB1 intron 1 was identified. For final statistic, the sheep carrying VM-resistant TMEM154 diplotypes were removed and a McNemar's test with a matched pairs experimental design was conducted. Consequently, it was identified for the first time that the $2 \mathrm{bp}$ de/variant is a genetic risk factor for VM ( $p$-value, 0.002 ; chi-square, 9.3; odds ratio, 2.9; statistical power >0.93) in dominant model. Thus, negative selection for 2 bp de/ variant could decrease VM infection risk in Turkish sheep.

\section{Introduction}

Small ruminant lentiviruses (SRLVs) such as caprine arthritis encephalitis virus (CAEV) in goats and Visna/maedi virus (VMV) in sheep cause prevalent chronic infections across the globe. The infection results in multisystemic inflammation like arthritis, mastitis, lymphadonopathy, interstitial pneumonia, meningoencephalitis, etc. SRLV infections are mainly characterized by an insidious onset, slow disease progression, and ultimate fatality. Both viruses (CAEV and VMV) are similar in their genetic structure that paves the way for the cross-species infection transmission between sheep and goat ${ }^{1-3}$.

Visna/maedi (VM) has been reported in Europe ${ }^{4,5}$, North America ${ }^{6}$, South America ${ }^{7}$, Asia ${ }^{8}$, and Africa ${ }^{9,10}$. Only New Zealand and Australia are yet VM free, nonetheless, these countries are suffering from CAEV ${ }^{11}$. VM was first reported in Turkey in $1987^{12}$. Regardless of sampling methods, successive reports showed that VM seroprevalence was from $2.7-77.9 \%{ }^{13-15}$. In a recent serosurvey on randomly sampled seven native Turkish breeds and four crossbreds $(n=2266)$, it was demonstrated that the breed-level mean seroprevalence was $12.7 \%$ ranging from $2-83.1 \%$. In the same study, 13 out of 16 flocks were seropositive, and the flock level prevalence was $81.3 \%{ }^{16}$.

There is no effective vaccine against VM as of the present. Once the animal acquires the VM virus, it will remain as a life long reservoir of the virus due to the persistency of infection ${ }^{1}$. Several studies reported indirect production losses caused by VM through low conception rates, reduced milk production, lower birth or weaning weight of lambs given by infected ewes ${ }^{17}$. On the other side, death or premature culling of infected animals are direct production losses ${ }^{18}$. The estimated VM associated production loss was up to $20 \%$ in the USA ${ }^{19}$ and $40 \%$ in UK ${ }^{17}$.

Testing and culling VM positive ewes and their lambs, the requirement of extra facilities to keep positive sheep until culling, restocking ewes, etc., makes the infection eradication efforts much more difficult in terms of cost and labor ${ }^{20}$. Moreover, screening and culling eradication strategy for VM does not guarantee long-lasting infection-free flocks as flocks will remain open to a new infection at any time. A typical example of such case is a field trial in 1979, where thirteen VM affected commercial flocks with $17 \%$ mean seroprevalence were subjected to screening and culling program for every six months. After two years, at the 5, 6, and 7th screening, all flocks were found seronegative. However, at the 8th test, three VM seropositive sheep were found where the exact source of the infection remained unclear ${ }^{21}$.

The search for ways to implement effective selective breeding strategies against lethal and chronic diseases like VM has been gaining increased attention worldwide. With various statistical power, several candidate loci such as ovar-DRB $7^{22,23}, C C R 5^{24}, D P P A 2 / D P P A 4$ and $S Y T L 3^{25}, Z N F 389^{26}$, and $T L R 9^{27}$ were reported to be associated with the VM occurrence. Moreover, a case-control matched pairs experimental design revealed two haplotypes (haplotypes 1 and 4) in Exon2 region of TMEM154 gene having a major effect according to the recessive model on reducing susceptibility to VM in North American sheep ${ }^{28}$. Subsequently, studies have confirmed this association in North American ${ }^{29,30}$, German, Iranian ${ }^{31,32}$, and Turkish sheep ${ }^{16}$. The frequencies of the protective TMEM154 haplotypes, however, were reported to be significantly low or absent in some indigenous Turkish and Iranian sheep ${ }^{16,32}$. Although TMEM154 has a key role in VM resistance/susceptibility, there could be other possible co-receptors that affect VM occurrence. In case the possible co-receptors are detected; they could be used in selective breeding for VM in indigenous sheep breeds.

Previous attempts by our team to investigate the association between some previously reported VM associated genes, i.e., CCR5, ZNF389, $T L R 9$ and VM serostatus did not reveal any significant result when a case-control experimental design was implemented (unpublished data). Here we aimed to investigate the possible link between Ovar-DRB1 and VM serostatus. Ovar-DRB1 was reported to be associated with VM in two independent studies ${ }^{22,23}$. DRB1 is a MHC class II gene and plays a crucial role in recognizing peptides of pathogens and presenting them to the T cells. The function of DRB1 gene in the mammalian immune system is well studied ${ }^{33,34}$. In the present study, a case-control matched pairs experiment was carried out to investigate the possible association between Ovar-DRB1 variants and serostatus of VM in Turkish sheep.

Page $2 / 11$ 


\section{Results}

Sequences obtained by using standart and universal M13 tagged primers showed $100 \%$ concordance. A total number of sixty-five $D R B 1$ Exon 2 haplotypes were estimated across all breeds. 10:01, 13:01, 09:02, and 08:01 were the most prevalent haplotypes which occurred at an overall frequency greater than 0.05 , i.e., $0.19,0.12,0.09$, and 0.09 , respectively. Some of the haplotypes were breed-specific, whereas some haplotypes $(n=21)$ found as single copy across all sample set. Regarding the haplotypes which were detected at least two times across all breeds, broad haplotype diversity was observed in Kivircik (31), Bandirma (29), Merino (20), and Imroz (13; Fig. 1; Supplemental table S1). The principal components (PC) plot for PC1 (21.8\%) versus PC2 (20.3\%) demonstrated that SBA crosses, Hampshire crosses, Ramlic and native Chios were slightly clustered regarding DRB1 genotypes according to breeds and the most distinct breed was Imroz (Fig. 2).

Alongside DRB1 Exon 2 haplotypes, a 2 bp deletion mutation (2 bp del) was identified at the Intron 1 region of $D R B 1$ gene, just 11 bp upstream of Exon 2 (Fig. 3). Deleted nucleotides are "CG" and the exact genomic position of the deletion mutation is 20:27300971-72 (NC_040271.1). High Linkage Disequilibrium (LD) was observed between the 2 bp de/ mutation and 13:01 haplotype (D' value, 0.984; r-squared, 0.836), moreover, the highest LD was identified between the 2 bp de/ mutation and detected haplogroup 13 (haplotypes 13:01 and 13:03; D' value, 0.984; r-squared, 0.903; Fig. 4). Haplotype 13:02 was not informative for LD analysis as it was detected as only one copy. The 2 bp del mutation was not found in Karacabey merino, Ramlic, and Hampshire crosses. The frequencies of the $2 \mathrm{bp}$ del mutation in Chios, Imroz, Kivircik, Bandirma, and SBA was observed at a range of 0.03 (Imroz) to 0.42 (SBA; Table 1).

Table 1

Frequencies of the $2 \mathrm{bp}$ de/ mutation in 151 matched pairs

panel.

\begin{tabular}{|llll|}
\hline Breed & Situation & n & 2 bp del \\
\hline Chios & native & 4 & 0.250 \\
\hline Imroz & native & 32 & 0.031 \\
Kivircik & native & 94 & 0.144 \\
Merino & improved breed & 34 & - \\
\hline Ramlic & improved breed & 2 & - \\
\hline SBA & research flock & 6 & 0.417 \\
\hline Hampshire crosses & research flock & 6 & - \\
\hline Bandirma & research flock & 124 & 0.181 \\
\hline & Overall & 302 & 0.134 \\
\hline
\end{tabular}

A McNemar's test was performed over 151 matched pairs to investigate the correlation between the $2 \mathrm{bp}$ del mutation or the most prevalent haplotypes (10:01, 13:01, 09:02, and 08:01) and VM serostatus (Supplamental table S2). Both recessive and dominant models were tested. In McNemar's test, the sum of the discordant pairs $(1 ; 0$ and $0 ; 1)$ is expected to be higher than 25 for statistical significance. Accordingly, all of the tested variants were observed to have higher than 25 discordant pairs for the dominant model but not for the recessive model. A statistically significant correlation was observed between VM serostatus and haplotype 13:01 and the 2bp de/ mutation according to the dominant model (Table 2), among these two variants, however, the most significant association was detected between the 2 bp de/ mutation and VM serostatus (exact $p$-value, 0.002; chi-square, 9.38; odds ratio, 2.57; $\mathrm{Cl} 95$ ) upon computing the dominant model. Statistical power analysis was performed using real sample size (151 matched pairs) and percentage of discordant pairs (33.1\%). Hence, the statistical power of the first analysis was calculated to be $>0.91(p<0.05)$. 
Table 2

McNemar's statistics of DRB1 haplotypes and $2 \mathrm{bp}$ del mutation

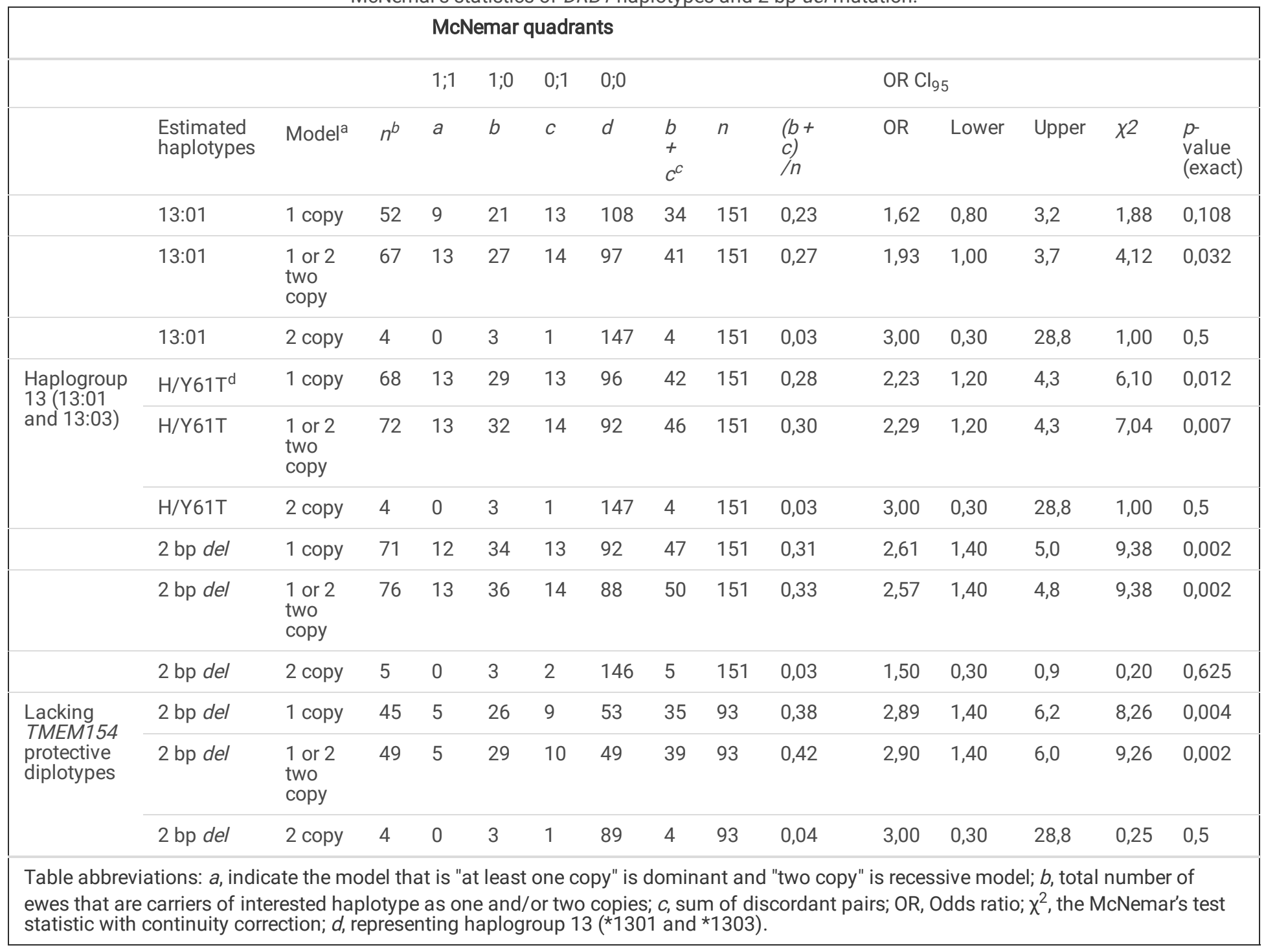

Besides the high LD between the 2 bp del and haplotype 13:01, it was identified that Histidine $(H)$ or Tyrosine $(Y)$ substitution to Threonine ( $T$ ) at codon 61 (H/Y61T) was also linked to both 2 bp del and 1301 haplotype (Fig. 4). Among 124 ovine DRB1 haplotypes deposited in Immuno Polymorphism Database (IPD), the 61T amino acid variant is specific to 13:01, 13:02, 13:03, 05:01:01, 05:02:01, 05.03:01, 26:01, and 29:01 haplotypes of which only 13:01, 13:02, 13:03, and 26:01 of were detected in the present study with the overall frequencies of $0.12,0.003$, 0.007, and 0.005, respectively. Another LD analysis was performed over the $2 \mathrm{bp}$ de/ mutation and the haplogroup 13 (haplotypes 13:01, 13;02, and 13:03) which are carrier of 61T missense variants. Due to the haplotype 26:01 was detected as three copy across all breed and neither those were not carrier of $2 \mathrm{bp}$ de/ variant, this haplotype was excluded this analysis. Eventually, almost a perfect LD was observed between the 2 bp de/ variant and the 61T amino acid carrier haplogroup 13 (D' value, 0.985; r-squared $\geq 0.903 ;$ Fig. 4). Thus, second McNemars's test for the haplogroup 13 with 46 discordant pairs revealed a similar association with the 2 bp de/mutation (exact $p$-value, 0.007 ; odds ratio, 2.3 ) in dominant model (Table 2).

Our case-control matched pairs panel was also available for TMEM154 genotypes which were defined to have a major effect for the recessive model on VM resistance/susceptibility ${ }^{16,28-32}$. Finally, to test the relative protection of the wild type DRB1 genotype compared to the 2 bp del variant in the absence of the protective effect of TMEM154, these matched pairs was sorted and analyzed again. Briefly, if at least one member of a pair is a carrier of protective TMEM154 diplotypes $(1 ; 1,1 ; 4$, or 4;4), these pairs were removed from the data set, thus, 93 matched pairs lacking the protective TMEM154 diplotypes remained (Supplemental table S3). Consequently, the third McNemar's test for correlated proportion was performed on this data set with 39 discordant pairs $(1 ; 0$ and 0;1). It was determined that 2 bp de/mutation was still significantly associated with increased susceptibility, and the wild type ones were associated with relative resistance to VM despite lacking the protective TMEM154 diplotypes (exact $p$-value, 0.002; chi-square, 9.26; odds ratio, 2.9; $\mathrm{Cl} 95$ ). The statistical power of this analyzis was greater than 0.93 (odds ratio, 2.9; $\mathrm{Cl} 95 ; p<0.05$ ). According to our results, the 2 bp de/ mutation in DRB1 Intron1 was identified as a genetic risk factor 
in dominant model, i.e., having one or two copies of $2 \mathrm{bp}$ de/mutation was found to increase the risk of contracting the VM virus by 2.9 fold (Table 2).

\section{Discussion}

As in many other mammalians, there are several identified ovine DRB1 gene variants. According to IPD records, 124 ovine $D R B 1$ alleles or subtypes have been deposited so far. In the present study, a broad genetic diversity in Exon 2 region of DRB1 gene was identified in Turkish sheep. There were 44 different haplotypes detected in at least two times (Supplemental table S1). The highest relative allelic diversity (the number of the detected different alleles over total alleles in each breeds) was in Chios breed (0.625) and the lowest in Kivircik breed (0.176).

Exon 2 region of the ovar DRB1 gene has gained a great attention of researchers due to having a broad genetic diversity, and its key role in immune defense. Previously, the association between various DRB1 alleles and different diseases such as Cystic Echinococcosis ${ }^{35}$, faecal egg count of gastrointestinal nematodes ${ }^{36,37}$ have been reported. The association between DRB1 gene and resistance/susceptibility to VM have also been reported in two different studies. In the first study, it was found that DRB1 haplotypes 04:03 and 07:12 were significantly associated with lower provirus levels of the ovine progressive pneumonia ${ }^{22}$, which is the counterpart of VM in North America, and in the second study, it was reported that the haplotype 03:25 was associated with the susceptibility against $\mathrm{VM}^{23}$. However, these reported haplotypes were not detected in the present study.

According to our findings, strong LD was detected between $2 \mathrm{bp}$ de/variant and 61T amino acid substitution which was found in 13:01 and 13:03 haplotypes in our genotype panel. To further investigate, fifty Ovar-DRB1 sequences consisting of 2 bp delvariant in intron 1 were obtained from Genbank (Accession numbers: MG000511.1, MG000512.1, MG000515.1, MG000516.1, MG000518.1, MG000538.1 to 552.1, and MG000555.1 to 586.1). All the sequences were belong to the Djallonke and Sahelian native sheep breeds of Ghana in West Africa. These sequences were searched using BLAST on IPD server, and the results revealed that all of these sequences were compatible with the haplotype 13:02 (D' value, 1; r-squared, 1). Archaeological evidence and retrovirus integration analyses suggest that selection for desired traits common to modern sheep first began in Fertile Crescent including the Anatolia region of Turkey, and spread to Africa, Europa, and other parts of Asia ${ }^{38}$. All the native breeds in the present study were carriers of the $2 \mathrm{bp}$ de/mutation, but improved breeds by backcrossing (Karacabey merino and Ramlic) were not. Other two research flocks, Bandirma which was bred from native Kivircik breed and SBA crosses, were also carriers of this mutation. When the Genbank records and our finsings are evaluated together; it can be inferred that having been found in African and Turkish native breeds, 2 bp deletion is an ancient mutation and perfectly linked to both haplogroup 13 (13:01, 13:02, and 13:03) and 61T amino acid variation. Furthermore, it could be speculated that $61 \mathrm{~T}$ amino acid substitution, either alone or in combination with other codons, may be the causative variant for increased susceptibility against VM infection. However, case control studies are required with other $61 \mathrm{~T}$ carrier haplotypes ie. 05:01:01, 05:02:01, 05.03:01, 26:01, and 29:01 to strengthen this hypothesis. Furthermore, the LD status between 2 bp del and $61 \mathrm{~T}$ amino acid variant on these haplotypes remained ambiguous. Despite these limitations, it was clearly demonstrated that 2 bp del mutation in the intron 1 of the ovine DRB1 is a strong predictor for the $61 \mathrm{~T}$ amino acid variant in haplogroup 13 , and significantly associated with increased susceptibility against VM.

In a recent study, the VM resistant TMEM154 haplotypes in Turkish native sheep breeds were detected either at a very low frequency or complately absent (0 to 0.12 ) when compared to the improved breeds by backcrossing ${ }^{16}$. A similar observation was made in Iranian native sheep breeds ${ }^{32}$. Hence, selective breeding regarding protective TMEM154 genotypes might be a long lasting process to improve herd level genetic resistance to VM for native breeds. Alternatively, introgression or gene editing technology, i.e., CRISPR might be required for the native breeds lacking resistant TMEM154 haplotypes. Otherwise, $2 \mathrm{bp}$ de/mutation and linked H/Y61T amino acid substitution was found a range of

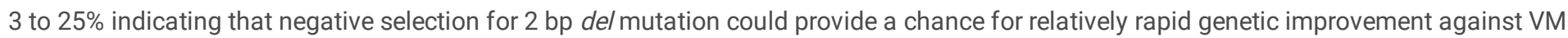
disease for some native sheep.

From those 151 matched pairs panel, 21 ewes were carriers of susceptible DRB1 2 bp de/ variant and protective TMEM154 diplotypes (1;1, 1;4, or 4;4) at the same time. According to the previous reports, TMEM154 protective diplotypes provide approximately 3-fold protection to VM, thus, it could be expected that only $1 / 4$ of these 21 ewes could be VM positive. Ten of these ewes, however, were VM positive which might be indicative that $2 \mathrm{bp}$ de/ variants have been limiting the protective effect of the TMEM154. Thus, further genetic improvement could be achieved using combination of these two genetic markers (DRB1 and TMEM154) for selective breeding of both native and improved sheep breeds.

Genotyping of MHC II locus, including DRB genes is highly problematic due to the excessive variation in a relatively short exon often causes sequence reading errors. Therefore, bidirectional sequencing is needed to reduce bias. In fact, bidirectional sequencing does not guarantee error-free genotyping, therefore, it is highly recommended to clone sequences for increased precision while genotyping. However, detection of 2 bp de/ variation could be much easier and cost effective with availability of genotyping techniques such as Allele Specific PCR (AS-PCR), 
Restriction Fragment Length Polymorphism (RFLP), Single Strand Conformationel Polymorphism (SSCP), or Real-Time PCR, etc. Besides, genotyping of $2 \mathrm{bp}$ de/ variant using such techniques might result in much fewer genotyping errors when compared to the exon sequencing.

In conclusion, this is a pioneering study in the identification of the association between the 2 bp de/ variation in ovine $D R B 1$ intron 1 and VM serostatus in the presence and/or absence of the protective effect of the major gene TMEM154. The protective haplotypes of TMEM154 were previously detected at a high frequency in improved breeds such as Karacabey merino and Ramlic, and for native breeds they were in very low frequencies or absent. Conversely, 2 bp de/variant of the $D R B 1$ gene having a strong association with increased susceptibility to VM was found in many native Turkish breeds. Thus, considering the prevalence of VM in some native breeds, it is highly reasonable to take the $D R B 12$ bp de/ variant into account for selective breeding to obtain infection-free sheep flocks.

\section{Methods}

\section{Animals}

In this study, three indigenous Turkish breeds (Chios, Imroz, and Kivircik), two improved breeds (Karacabey Merino and Ramlic), and three composite breeds (Blackhead merino crosses-SBA, Hampshire crosses, and Bandirma) were considered. Chios, Imroz, and Kivircik are ancient breeds that are well-adapted to their environment for thousands of years. Karacabey merino was improved by backcrossing in the 1940 's in Sheep Breeding and Research Institute (SRI), whereas Ramlic was improved in the 1960's by the Turkish Ministry of Agriculture and Forestry. Both breeds have been closed for backcrossing for more than 30 years. The composite breeds: SBA, Hampshire, and Bandirma have been reared as research flocks at SRI. Ewes for the study were selected according to their serostatus, age, breed type, and flock from the 2017-VM serosurvey cohort of 2266 ewes of eleven flocks at six different locations ${ }^{16}$. To allow sufficient VM virus exposure and subsequent seroconversion, only the ewes that were two years or older ages were included (ages ranging from two to eight). Thus, a total number of 302 ewes (151 cases and 151 controls) were included in the study. All methods were performed in accordance with the relevant guidelines and regulations.

\section{Genetic Analysis}

Genomic DNA was extracted from peripheral whole blood with EDTA using commercial spin-column DNA extraction kits (GeneAll, Seoul, Korea) according to the manufacturer's instructions. Polymerase chain reaction (PCR) was carried out to amplify Exon 2 region of Ovar-DRB1 gene using previously designed primers (DRB1_330_F: ATTAGCCTCYCCCCAGGAGKC and DRB1_329_R: CACCCCCGCGCTCACCTCGCCGC) ${ }^{39}$. Amplified products overlap the last $54 \mathrm{bp}$ of $D R B 1$ Intron 1 and $270 \mathrm{bp}$ of the full sequence of Exon 2 . Standard Sanger sequencing analysis was performed as follows: pre-purification of PCR products by Exo-SAP incubation followed by chain termination reaction with BigDye ${ }^{\text {TM }}$ Terminator v3. 1 kit, post-purification with ethanol precipitation protocol, and capillary electrophoresis on $A B 3500$ genetic analyzer were performed sequentially. All samples were sequenced bidirectionally. Approximately 1/4 (28\%) from all samples were selected randomly and amplified separately using the same primers with universal M13 primer tags (M13_F: GTAAAACGACGGCCAGT and M13_R: AACAGCTATGACCATG) and sequenced bidirectionally to validate the obtained sequences and check primers binding sites.

\section{Statistical analysis}

Chromatograms were visualized using FinchTV v1.4.0 software (Geospiza, Inc) and aligned by MEGA v6.0 software ${ }^{40}$. DRB1 Exon 2 haplotypes were estimated using Phase v2.1 algorithm ${ }^{41}$ and identified by Basic Local Alignment Search Tool (BLAST) in both National Center for Biotechnology (NCBl; https://www.ncbi.nlm.nih.gov/) and Immuno Polymorphism Database (IPD; https://www.ebi.ac.uk/ipd/). Linkage disequilibrium (LD) analysis was performed using Haploview v4.2 $20^{42}$.

Host genotype and disease resistance/susceptibility association study requires the control of confounding factors such as variations in exposure intensity, exposure duration and other environmental factors like climate, herd management, herd density, access to the pasture, etc. Furthermore, it is required to increase statistical power to reduce false positive results potentially caused by the population stratification phenomenon. Therefore, association analysis was performed according to case-control matched pairs experimental design. A total of 151 matched pairs (151 cases and 151 controls) were constructed according to age, breed type, and flock. Briefly, a seropositive ewe was randomly matched with a seronegative one from the same flock, same breed type, and same age group (Table 3). Thus, pairs were assigned to be (1;1), $(1 ; 0),(0 ; 1)$, and $(0 ; 0)$ i.e. both case and control are carriers of genetic risk/protective factors $(1 ; 1)$, the case is the carrier of genetic risk/protective factors, but control is not $(1 ; 0)$, the case is not a carrier of genetic risk/protective factors, but control is $(0 ; 1)$, and both case and control are not carriers of genetic risk/protective factors $(0 ; 0)$. In this experimental design, the higher rate of the discordant pairs $(1 ; 0$ and $0 ; 1)$ provides higher statistical power. The age information was not available for a small proportion of matched pairs panel (5.3\%). Thus, these individuals were matched according to breed type and flock. A McNemar's test ${ }^{43}$ for correlated proportions was performed using these matched pairs. To determine the statistical power of the experimental design, a power analysis was conducted using the $\mathrm{G}^{\star} \mathrm{Power}$ software ${ }^{44}$. 
Table 3

Age and breed composition of the matched pairs panel.

\begin{tabular}{|c|c|c|c|c|c|c|c|c|c|c|}
\hline Age & Chios & Imroz & Kivircik & Karacabey Merino & Ramlic & SBA & Hampshire crosses & Bandirma & Total & $\%$ \\
\hline 8 & 0 & 0 & 0 & 0 & 0 & 0 & 0 & 10 & 10 & 3.3 \\
\hline 7 & 0 & 0 & 18 & 6 & 0 & 0 & 0 & 32 & 56 & 18.5 \\
\hline 6 & 0 & 10 & 24 & 6 & 2 & 2 & 4 & 26 & 74 & 24.5 \\
\hline 5 & 0 & 12 & 18 & 4 & 0 & 0 & 0 & 30 & 64 & 21.2 \\
\hline 4 & 0 & 4 & 14 & 6 & 0 & 0 & 2 & 20 & 46 & 15.2 \\
\hline 3 & 0 & 4 & 8 & 10 & 0 & 4 & 0 & 6 & 32 & 10.6 \\
\hline 2 & 0 & 2 & 0 & 2 & 0 & 0 & 0 & 0 & 4 & 1.3 \\
\hline na & 4 & 0 & 12 & 0 & 0 & 0 & 0 & 0 & 16 & 5.3 \\
\hline Total & 4 & 32 & 94 & 34 & 2 & 6 & 6 & 124 & 302 & 100 \\
\hline$\%$ & 1.3 & 10.6 & 31.1 & 11.3 & 0.7 & 2.0 & 2.0 & 41.1 & 100 & \\
\hline
\end{tabular}

\section{Declarations}

\section{Acknowledgements}

We thank Veterinarian Ahmet IPçi and Veterinary technician Süleyman ÖZDEMIR for their important helps to field coordination and sampling.

\section{Funding}

Funding for this research was provided by the Republic of Turkey Ministry of Agriculture and Forestry, General Directorate of Agricultural Research and Policies (TAGEM) (Project number: TAGEM/HAYSÜD/15/A01/P02/02-02).

\section{Competing interests}

The authors declare no competing interests.

\section{Author Contributions}

Y.Y. conceived and designed the study, conducted the statistical analysis, Y.Ö and V.B performed quality controls of genomic data and conducted haplotype construction, R.A and M.K conducted laboratory experiments, E.Y.T and C.Ü. reviewed the manuscript and assisted in data interpretation. All authors read and approved the manuscript.

\section{Ethics declarations}

All animal procedures in the study were reviewed and approved by the local ethics committee of Sheep Breeding and Research Institute (Approval Number: 1282412), and the authors complied with the ARRIVE guidelines.

\section{References}

1. Pépin, M., Vitu, C., Russo, P., Mornex, J-F. \& Peterhans, E. Maedi visna virus infection in sheep: a review. Vet Res. 29, $341-367$ (1998).

2. Shah, C. et al. Direct evidence for natural transmission of Small-Ruminant Lentiviruses of subtype A4 from goats to sheep and vice versa. J Virol. 78, 7518-7522 (2004).

3. Reina, R. et al. Prevention strategies against small ruminant lentiviruses: an update. Vet J. 182, 31-37 (2009).

4. Hüttner, K., Heyne, H. \& Heim, D. Impact of flock segregation according to the Maedi-Visna status on reproduction and lamb rearing - a field study in Mecklenburg-Vorpommern Berliner und Münchener Tierärztliche Wochenschrift. 130, $490-493$ (2017).

5. Ogden, N., Davies P. \& Lovatt, F. Dealing with maedi visna in UK sheep flocks In Practice 41, $321-328$ (2019).

6. Heinrichs, R., Wilkins, W., Schroeder, G. \& Campbell, J. Prevalence of Maedi-visna in Saskatchewan sheep: Can Vet J. 58, 183-186 (2017). 
7. Alves, S. M. et al. Seroepidemiological study of maedi-visna in sheep in Ceara, Rio Grande do Norte, Paraíba, and Sergipe States Semina. Ciências Agrárias. 39, 2017-2028 (2018).

8. Oguma, K. et al. Isolation of Maedi / Visna Virus from a Sheep in Japan. J Vet Med Sci. 76, 211-218 (2014).

9. Ayelet, G., Roger, F., Tibbo, M. \& Tembely, S. Survey of Maedi-Visna (MV) in Ethiopian Highland Sheep. Vet J. 161, 208-210 (2001).

10. Tabet, E., Tlaige, R., El Hage, J. \& Abi-Rizk, A. The occurrence of Maedi-Visna Virus in Lebanon Rev Sci Tech. 36, 899-903 (2017).

11. Gomez-Lucia, E., BaVrquero, N. \& Domenech, A. Maedi-Visna Virus: Current Perspectives. Vet Med (Auckl). 21, 11-21 (2018).

12. Girgin, H., Aydın, N., Yonguç, A. D., Aksoy, E. \& Çorak, R. Ve şimdi koyunların Viral Maedi-Visnası Türkiye'de Etlik Vet Mikrob Derg. 9 , 20 (1987).

13. Burgu, İ. et al. Türkiye'de Visna-Maedi enfeksiyonunun serolojik olarak araştırılması. A Ü Vet Fak Derg. 37, 538-553 (1990).

14. Yavru, S., Şimşek, A., Bulut, O. \& Kale, M. Serological investigation of Maedi-Visna Virus infection in sheep in Konya region. Eurasian Journal of Veterinary Sciences 28, 142-48 (2012).

15. Muz, D. et al. First molecular characterization of visna/maedi viruses from naturally infected sheep in Turkey. Arch Virol. 158, 559-70 (2013).

16. Yaman, Y. et al. Association of TMEM154 variants with visna/maedi virus infection in Turkish sheep Small Rum Res. 177, 61-67 (2019).

17. Benavides, J. et al. Impact of maedi-visna in intensively managed dairy sheep. Vet J. 197, 607-612 (2013).

18. Peterhans, E. et al. Routes of transmission and consequences of small ruminant lentiviruses (SRLVs) infection and eradication schemes. Vet Res. 35, 257-74 (2004)

19. Keen, J. E., Hungerford, L. L., Littledike, E. T., Wittum, T. E. \& Kwang, J. Effect of ewe ovine lentivirus infection on ewe and lamb productivity Prev Vet Med. 30,155-169 (1997).

20. Fisher, J. W. \& Menzies, P. I. Cost of a Maedi Visna flock certification program and the changes in productivity and economic return. Sheep \& Goat Research Journal. 20, 17-24 (2005).

21. Houwers, D. J., Schaake, J. \& de Boer, G. F. Maedi-visna control in sheep II. Half-yearly serological testing with culling of positive ewes and progeny. Vet Microbiol. 9, 445-451 (1984).

22. Herrmann-Hoesing, L., White, S. N., Mousel, M. R., Lewis, G. S., Knowles, D. P. Ovine progressive pneumonia provirus levels associate with breed and Ovar-DRB1. Immunogenetics. 60, 749-758 (2008).

23. Larruskain, A. et al. MHC class II DRB1 gene polymorphism in the pathogenesis of Maedi-Visna and pulmonary adenocarcinoma viral diseases in sheep Immunogenetics. 62, 75-83 (2010).

24. White, S. N., Mousel, M. R., Reynolds, J. O., Lewis, G. S. \& Herrmann-Hoesing, L. M. Common promoter deletion is associated with 3.9-fold differential transcription of ovine CCR5 and reduced proviral level of ovine progressive pneumonia virus. Anim Genet 40, 583-9 (2009)

25. White, S. N. et al. Genome-wide association identifies multiple genomic regions associated with susceptibility to and control of ovine lentivirus. PLoS One. 7, e47829 (2012).

26. White, S. N., Mousel, M. R., Reynolds, J. O., Herrmann-Hoesing, L. M. \& Knowles, D. P. Deletion variant near ZNF389 is associated with control of ovine lentivirus in multiple sheep flocks Anim Genet. 45, 297-300 (2014).

27. Sarafidou, T. et al. Toll Like Receptor 9 (TLR9) polymorphism G520R in sheep is associated with seropositivity for small ruminant lentivirus. PLoS One. 8, e63901 (2013).

28. Heaton, M. P. et al. Reduced lentivirus susceptibility in sheep with TMEM154 mutations PLoS Genetics. 8, e1002467 (2012).

29. Leymaster, K. A., Chitko-McKown, C. G., Clawson, M. L., Harhay, G. P. \& Heaton MP: Effects of TMEM154 haplotypes 1 and 3 on susceptibility to ovine progressive pneumonia virus following natural exposure in sheep. J Anim Sci. 91, 5114-21 (2013).

30. Leymaster, K. A., Chitko-Mckown, C. G. \& Heaton, M. P. Incidence of infection in 39-month-old ewes with TMEM154 diplotypes "1 1," "1 3," and "3 3" after natural exposure to ovine progressive pneumonia virus. J Anim Sci. 93, 41-45 (2015).

31. Molaee, V., Eltanany, M. \& Lühken, G. First survey on association of TMEM154 and CCR5 variants with serological maedi-visna status of sheep in German flocks. Vet Res. 49, 36 (2018).

32. Molaee, V., Otarod, V., Abdollahi, D. \& Lühken, G. Lentivirus susceptibility in Iranian and German sheep assessed by determination of TMEM154 E35K. Animals. 9, 685 (2019).

33. Agrewala, J. N., Deacock, S., Jurcevic, S. \& Wilkinson, R. Peptide recognition by T-cell clones of an HLA-DRB1*1501/*0901 heterozygous donor is promiscuous only between parental alleles. Hum Immunol. 55, 34-38 (1997).

34. Gebe, J. A. et al. T cell selection and differential activation on structurally related HLA-DR4 ligands J Immunol. 167, 3250-56 (2001).

35. Shen, H., Han, G., Jia, B., Jiang, S. \& Du, Y. MHC-DRB1/DQB1 Gene polymorphism and its association with resistance/susceptibility to Cystic Echinococcosis in Chinese Merino Sheep. J Parasitol Res. 2014, 272601 (2014).

Page $8 / 11$ 
36. Charon, K. M., Moskwa, B., Rutkowski, R., Gruszczyńska, J. \& Świderek, W. Microsatellite polymorphism in DRB1 gene (MHC class II) and its relation to nematode faecal egg count in Polish heath sheep. J Anim Feed Sci. 11, 47-58 (2002).

37. Sayers, G., Good, B., Hanrahan, J. P., Ryan, M., Angles, J. M. \& Sweeey, T. Major histocompatibility complex DRB1 gene: its role in nematode resistance in Suffolk and Texel sheep breeds. Parasitology. 131, 403-409 (2005).

38. Chessa, B. et al. Revealing the history of sheep domestication using retrovirus integrations. Science. 324, 532-536 (2009).

39. Ballingall, K. T. \& Tassi, R. Sequence-based genotyping of the sheep MHC class II DRB1 locus. Immunogenetics. 62, 31-39 (2010).

40. Tamura, K., Stecher, G., Peterson, D., Filipski, A. \& Kumar S. MEGA6: Molecular Evolutionary Genetics Analysis version 6.0. Mol Biol Evol. 30, 2725-2729 (2013).

41. Stephens, M. \& Donnelly, P. A Comparison of Bayesian Methods for Haplotype Reconstruction from Population Genotype Data. Am J Hum Genet. 73, 1162-1169 (2003).

42. Barrett, J. C., Fry, B., Maller, J. \& Daly, M. J. Haploview: Analysis and visualization of LD and haplotype maps. Bioinformatics. 15;:263-265 (2005).

43. McNemar, Q. Note on the sampling error of the difference between correlated proportions. Psychometrika. 12,153-157 (1947).

44. Faul, F., Erdfelder, E., Buchne, A. \& Lang, A. G. Statistical power analyses using G*Power 3.1: tests for correlation and regression analyses. Behav Res Methods. 2009, 41; 1149-1160 (2009).

\section{Figures}

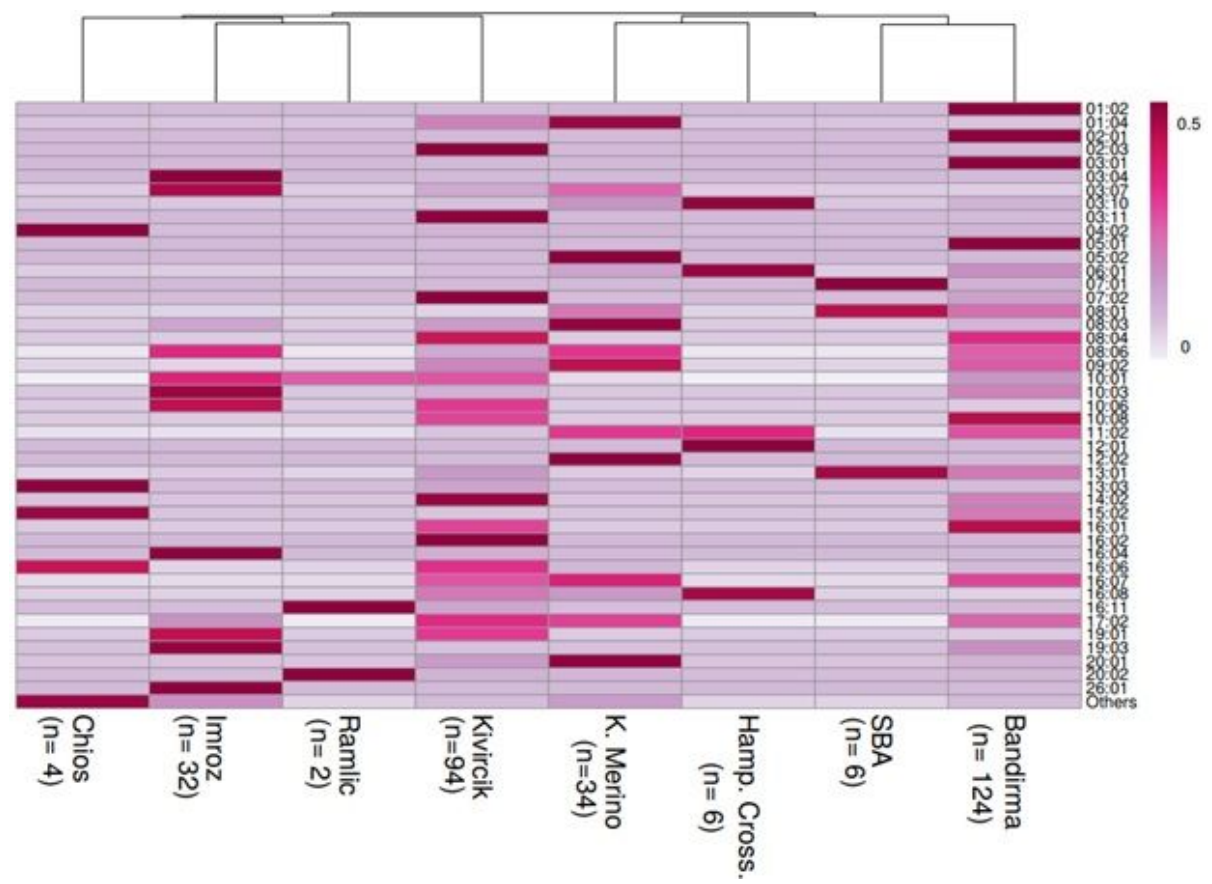

\section{Figure 1}

Distribution of DRB1 haplotypes according to breeds. 


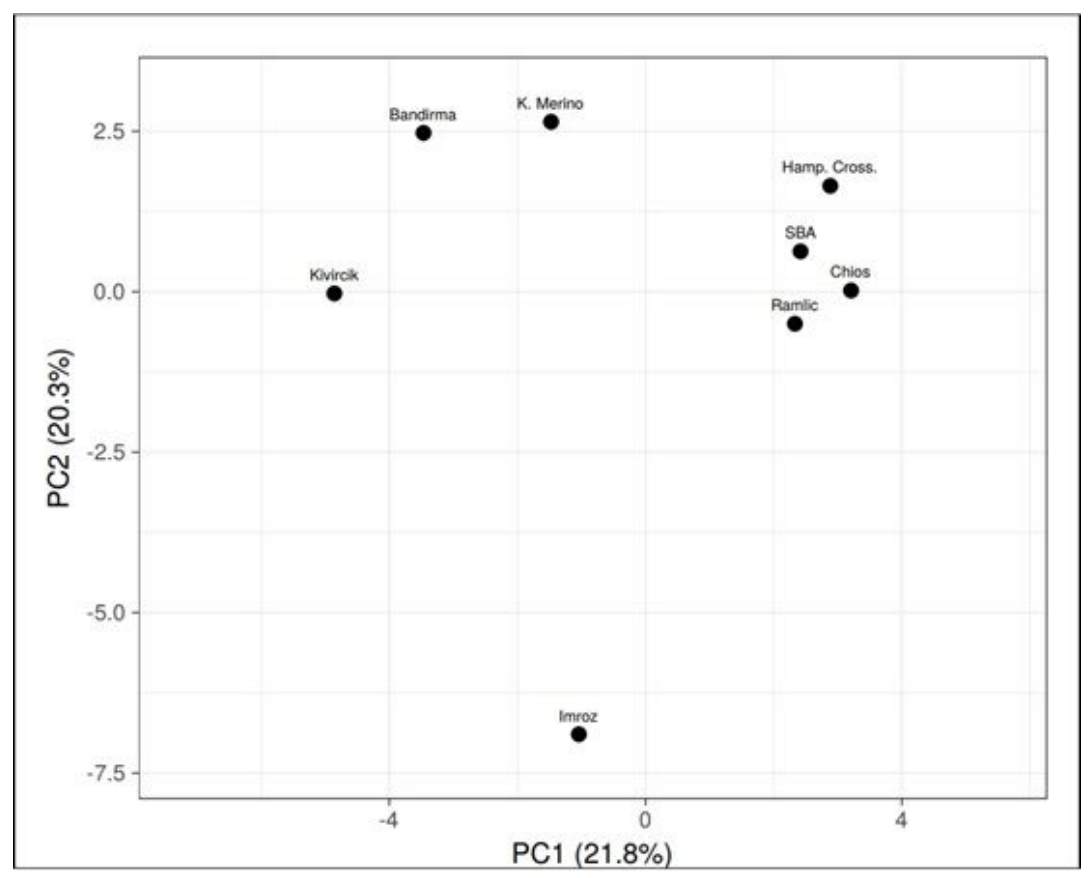

Figure 2

Principal components plot of DRB1 haplotypes according to breeds.

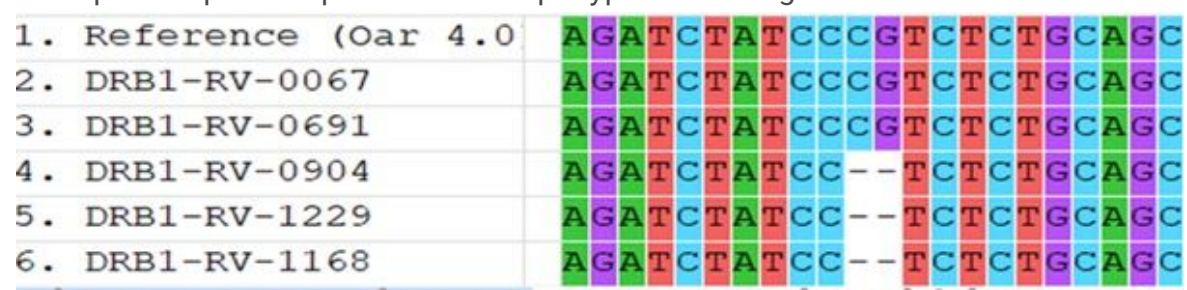

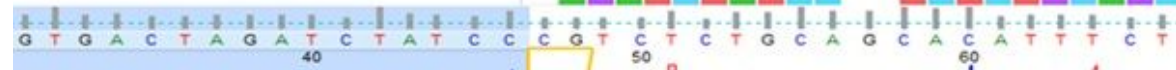

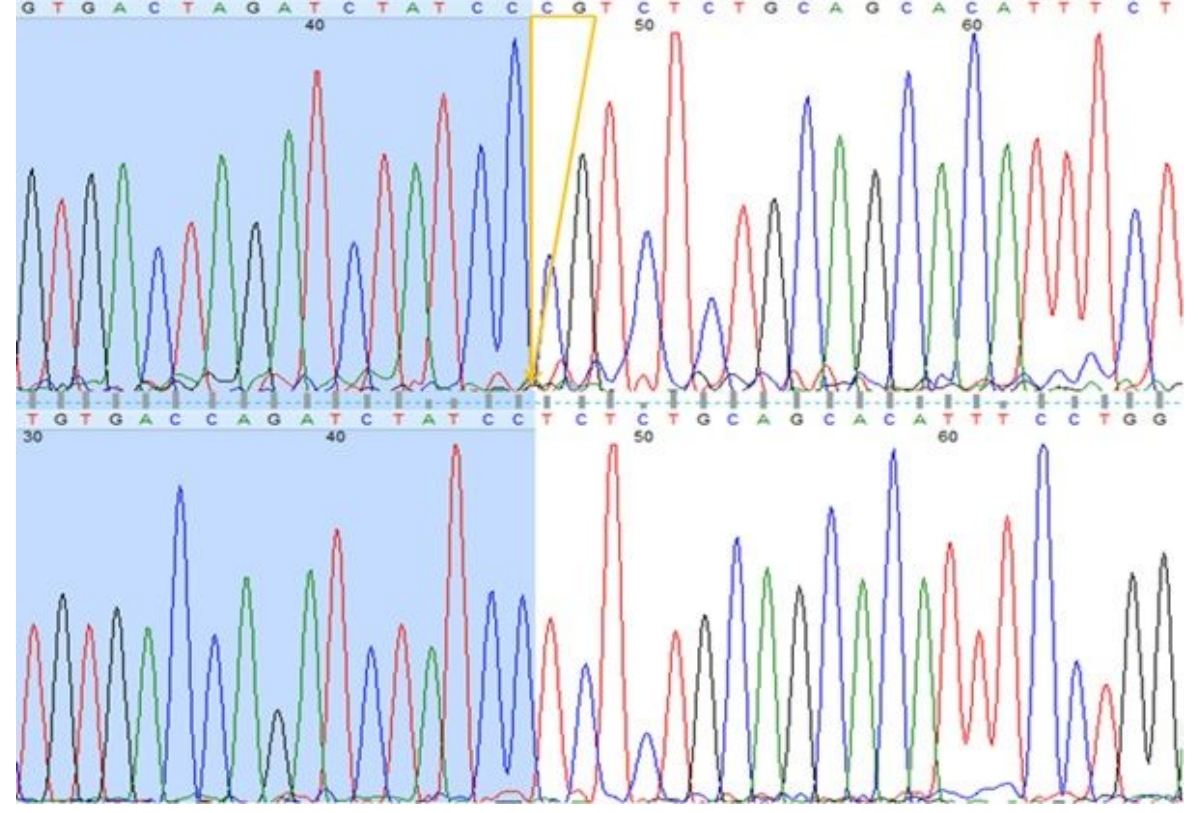

Figure 3

Alignment of DRB1 haplotypes and physical position of 2 bp del variant. Last three sequence in the alignment and the bottom figure in the chromatograms are deleted variants. 


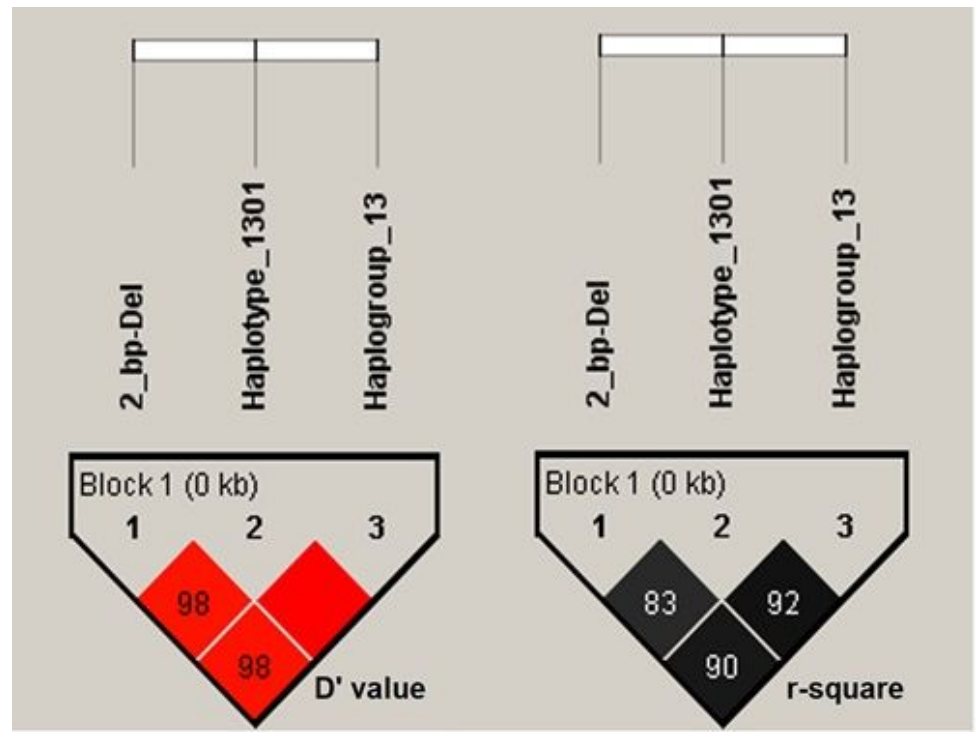

Figure 4

Linkage disequilibrium between 2 bp del variant, haplotype 13:01, and haplogroup 13.

\section{Supplementary Files}

This is a list of supplementary files associated with this preprint. Click to download.

- DRB1tableS1haplotypedistrubition.xlsx

- DRB1tableS2McNemarscasecontrolmatchedpairs.xlsx

- DRB1tableS3McNemarscasecontrolmatchedpairslackingTMEM154protectivediplotypes.xlsx 